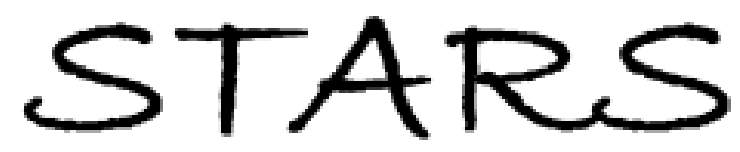

University of Central Florida

STARS

$1-1-2012$

\title{
Scaling analysis: Equivalence of convective and radiative heating of levitated droplet
}

\author{
Abhishek Saha \\ University of Central Florida \\ Saptarshi Basu \\ Ranganathan Kumar \\ University of Central Florida
}

Find similar works at: https://stars.library.ucf.edu/facultybib2010

University of Central Florida Libraries http://library.ucf.edu

This Article is brought to you for free and open access by the Faculty Bibliography at STARS. It has been accepted for inclusion in Faculty Bibliography 2010 s by an authorized administrator of STARS. For more information, please contact STARS@ucf.edu.

\section{Recommended Citation}

Saha, Abhishek; Basu, Saptarshi; and Kumar, Ranganathan, "Scaling analysis: Equivalence of convective and radiative heating of levitated droplet" (2012). Faculty Bibliography 2010s. 3227.

https://stars.library.ucf.edu/facultybib2010/3227

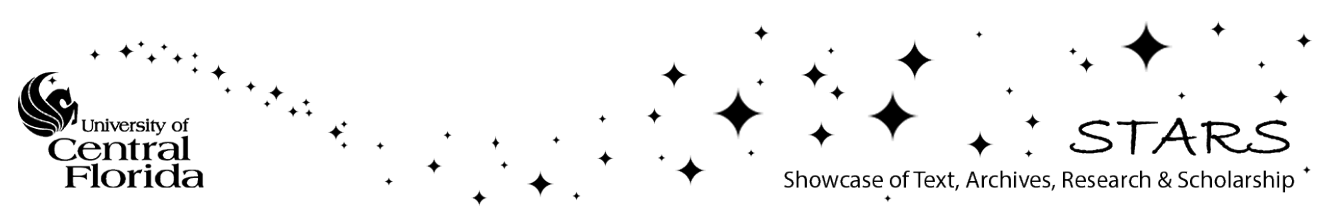




\section{Scaling analysis: Equivalence of convective and radiative heating of levitated droplet}

Cite as: Appl. Phys. Lett. 100, 204104 (2012); https://doi.org/10.1063/1.4720092

Submitted: 04 April 2012. Accepted: 03 May 2012. Published Online: 18 May 2012

Abhishek Saha, Saptarshi Basu, and Ranganathan Kumar

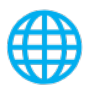

\section{ARTICLES YOU MAY BE INTERESTED IN}

Thermally induced secondary atomization of droplet in an acoustic field

Applied Physics Letters 100, 054101 (2012); https://doi.org/10.1063/1.3680257

Structural morphology of acoustically levitated and heated nanosilica droplet

Applied Physics Letters 97, 123106 (2010); https://doi.org/10.1063/1.3493178

Insight into morphology changes of nanoparticle laden droplets in acoustic field

Applied Physics Letters 102, 141602 (2013); https://doi.org/10.1063/1.4801502

\section{Applied Physics Reviews} Now accepting original research 


\title{
Scaling analysis: Equivalence of convective and radiative heating of levitated droplet
}

\author{
Abhishek Saha, ${ }^{1, a)}$ Saptarshi Basu, ${ }^{2, a)}$ and Ranganathan Kumar ${ }^{1, a), b)}$ \\ ${ }^{1}$ University of Central Florida, Orlando, Florida 32816, USA \\ ${ }^{2}$ Indian Institute of Science, Bangalore, India
}

(Received 4 April 2012; accepted 3 May 2012; published online 18 May 2012)

\begin{abstract}
This letter develops theoretical relationships for equilibrium timescale and temperature scale of a vaporizing droplet in a convective and a radiative environment. The transient temperature normalized by the respective scales exhibits a unified profile for both modes of heating. The analysis allows for the prediction of the required laser flux to show its equivalence in a corresponding heated gas stream. The theoretical equivalence shows good agreement with experiments across a range of droplet sizes. Simple experiments can be conducted in a levitator to extrapolate information in realistic convective environments like combustion and spray drying. (C) 2012 American Institute of Physics. [http://dx.doi.org/10.1063/1.4720092]
\end{abstract}

Evaporation of pure liquid droplet has been extensively studied in the last few decades. In a hot gas stream, it has widespread applications in gas turbines, thermal sprays, and spray drying of food, ceramics, and pharmaceutical products. Although the spray processes include droplet-droplet interactions and their effect on heat and mass transfer, the study of single droplet evaporation aids in the understanding of fundamental physics by visualization of breakup, precipitation, agglomeration kinetics, and structure formation. Owing to the difficulties in conducting droplet level investigation in an actual spray, the spray models are mostly developed and validated by single pendant droplet experiment.

Acoustic levitation is an alternative method to study micron-sized droplets without wall effects, which eliminates chemical and thermal contamination with surfaces. Acoustic levitator has been successfully used in material processing, crystal growth, pure and multicomponent droplet evaporation and protein synthesis. ${ }^{1-4}$ Experiments with droplet evaporation could be performed only at very low air flow rate and temperature so that the droplet can remain levitated. ${ }^{3,4}$ Alternatively, moderate to low laser heating of a levitated drop has been shown to be an effective way of droplet vaporization. This method allows us to understand the vaporization and agglomeration characteristics of new fluids, e.g., biofuel, ${ }^{4,5}$ nanosuspensions, ${ }^{6,7}$ and precursors ${ }^{8,9}$ economically without having to fabricate complicated optically accessible pre-mixers or combustion chambers.

Despite the advances made in droplet vaporization in a levitator using laser flux or by gas phase in convective environment, the two methods of heating have not been compared quantitatively so far, mainly due to the difficulties encountered in conducting experiments for the same size droplet under equivalent conditions and the lack of robustness of analytical techniques and the disparity in timescales that may exist between the two systems. We address four important issues pertaining to this problem. First, using scaling analysis and simplified droplet energy transport model (tran-

\footnotetext{
a) All authors contributed equally to this work.

b) Author to whom correspondence should be addressed. Electronic mail: ranganathan.kumar@ucf.edu.
}

sient and steady state regimes), we will show that the droplet wet bulb temperature and the time required to attain that temperature can be calculated analytically for both radiative and convective heating. Second, the normalized temperature data using the theoretical wet bulb temperature will be matched with the experimental data from the literature for both radiative and convective heating. Third, the normalized transient profiles from different sets of experiments will be shown to follow a single profile irrespective of the mode of heating. Fourth, the scaling analysis will be further extended to develop a connection between gas phase temperature in convective heating and effective laser flux in radiative heating for different fluids. A droplet in a convective environment vaporizes under the driving influence of temperature difference between the droplet surface and the gas field. In order to mimic similar conditions in the laser heating system in a levitator, the laser flux will be tuned to match the temperature difference. In addition, different fluids show a wide variation in absorption of laser irradiation. The equivalence (established through theoretical analysis) between the two modes of heat transfer will be validated by matching the experimental data for $125 \mu \mathrm{m}$ and $1000 \mu \mathrm{m}$ droplets from radiation experiments with the convective counterpart.

Theoretical models have been developed for droplet and spray evaporation. ${ }^{10,11}$ This involves solving the liquid phase transport equations and gas phase equations to calculate the heat and mass transfer. The effect of non-equilibrium modelling of droplet laden flows was also studied using LangmuirKnudsen formulations. ${ }^{12}$ Experimental studies of droplet evaporation have been largely restricted to dropsize over $1 \mathrm{~mm}$ (Refs. 12-14) in different fluids. A deviation from this norm is the series of articles published using optical techniques to measure evaporation characteristics of $100 \mu \mathrm{m}$ droplets. $^{15-17}$

Theoretical and experimental work in levitators ${ }^{2-8,18,19}$ have paved the way for conducting fundamental research in levitated functional droplet evaporation due to either natural drying or external heating. The shape and deformation of the droplet and its displacement from the pressure node may be used to calculate the acoustic streaming and the average distribution of Sherwood and Nusselt number around the 
droplet. $^{2}$ For comparable evaporation characteristics found in convective heating, levitated droplets can be irradiated with lasers. ${ }^{4-8}$ Laser heating of liquid droplets has also been studied by many authors albeit in non-levitator environment both experimentally and numerically. ${ }^{20-22}$

An evaporating droplet undergoing external heating (radiation or convection) exhibits two distinct regimes: the initial transient phase during which the bulk temperature increases and the steady state phase during which the temperature is constant (wet bulb temperature). The timescale and temperature scale associated with the external heating of a droplet is determined by both these phases. In the transient phase [Zone I in Fig. 1], the droplet temperature history is usually characterized by a balance between the energy supplied by the external heating source and the sensible enthalpy requirement for droplet temperature rise. Even though evaporation is present, it is not significant during this regime. However, the temperature increase implies a sharp upsurge of saturation vapor pressure which leads to increased evaporation rate. This shifts the balance, and the droplet now exhibits a steady state temperature (wet bulb limit) with all the input energy from the external heating source being utilized for vaporization [Zone II in Fig. 1]. The two regime lifecycle of a droplet is governed by thermo-physical properties such as latent heat, thermal conductivity, saturation vapor pressure, mass diffusivity, Reynolds number of the flow field, as well as the external heating conditions like laser flux (pure radiative heating) or the temperature of the surrounding gas phase (pure convective heating). The temperature scale and timescale are only relevant in the transient phase of the droplet [Zone I] though the steady state phase [Zone II] provides a limiting condition that enables us to uniquely ascertain the values of these scales. Similar methodology can be applied to both radiative and convective heating to establish the time and temperature scales. Using the same scales, one can also show the equivalence of radiative and convective heating.

The heat balance equation for a droplet under monochromatic radiation can be written as, ${ }^{20,21}$

$$
I_{\text {eff }} \cdot A_{s}=\frac{4}{3} \pi R^{3} \rho_{l} C_{p} \frac{d T_{s}}{d t}+Q_{\text {loss }}+\dot{m} h_{f g},
$$

$I_{\text {eff }}$ is the effective laser flux that is absorbed by the droplet (including effects of scattering losses and variation of absorption coefficients among various liquids), $\mathrm{A}_{\mathrm{s}}$, the surface area of the droplet exposed to the laser flux, $\mathrm{R}$, instantaneous droplet radius, $\rho_{1}$, liquid density, $\mathrm{C}_{\mathrm{p}}$, specific heat of the liquid, $\mathrm{h}_{\mathrm{fg}}$, the latent heat of the droplet, $\dot{m}$, the vaporization rate, $\mathrm{T}_{\mathrm{s}}$, the surface temperature. $\mathrm{Q}_{\text {loss }}$ represents the heat loss to the surrounding and is modeled as $Q_{\text {loss }}$ $=4 \pi R^{2} \cdot h_{\text {loss }} .\left(T_{s}-T_{0}\right)$, where $\mathrm{h}_{\text {loss }}$ is the convective heat transfer coefficient and $T_{0}$ is the ambient temperature and initial droplet temperature.

Neglecting vaporization until equilibrium [Zone II], the energy transport equation becomes

$$
I_{\text {eff }} \cdot A_{s}=\frac{4}{3} \pi R^{3} \rho_{l} C_{p} \frac{d T_{s}}{d t}+4 \pi R^{2} \cdot h_{\text {loss }} \cdot\left(T_{s}-T_{0}\right) .
$$

Since the droplet is asymmetrically heated from one side, the surface area irradiated is $A_{s}=2 \pi R^{2}$. It should be noted that for low to moderate laser flux, the asymmetric heating does not deviate significantly from the spherically symmetric heating. ${ }^{20}$ Although there is non-uniformity in the laser power and absorption coefficient along the droplet surface, Park and Armstrong analytically showed that for low to medium heating, the droplet temperature becomes more or less uniform. In our experiments, we do not observe any catastrophic breakup of droplets; hence, the effect of non-uniformity in absorption coefficient is not significant. IR images from the current experiments also display uniform temperature profile across the droplet surface. The heat loss can be calculated using the heat transfer analysis of a vaporizing droplet under acoustic levitation. Yarin et $a l^{3}$ showed that the Nusselt number (and Sherwood number) depends on acoustic streaming or acoustic intensity. The heat transfer coefficient is $h_{\text {loss }}=N u_{\text {str }} k_{\text {air }} / 2 R$. Equation (2) can be written as

$$
\frac{d T_{s}}{d t}=\frac{3 I_{\text {eff }}}{2 R \rho_{l} C_{p}}-\left(\frac{3 N u_{s t r} k_{\text {air }}}{2 R^{2} \rho_{l} C_{p}}\right) \cdot\left(T_{s}-T_{0}\right) .
$$

Assuming constant fluid properties and constant droplet diameter (till equilibrium or Zone II) and $\mathrm{T}_{\mathrm{s}}(\mathrm{t}=0)=\mathrm{T}_{0}$, Eq. (3) can be solved and the closed form solution is given by

$$
T_{s}(t)=T_{0}+\frac{C_{2-\mathrm{rad}}}{C_{1-\mathrm{rad}}}\left[1-\exp \left(-C_{1-\mathrm{rad}} t\right)\right],
$$

where $C_{1-r a d}=\frac{3 N u_{s t r} k_{\text {air }}}{2 R^{2} \rho_{l} C_{p}}$ and $C_{2-r a d}=\frac{3 I_{\text {eff }}}{2 R \rho_{l} C_{p}}$. To estimate the wet bulb temperature of the droplet in Zone II, it is assumed that the latent heat dominates sensible heat and the total heat flux is spent in vaporizing the droplet and heat loss to the surrounding. Thus, at equilibrium,

$$
I_{e f f} \cdot\left(2 \pi R^{2}\right)=4 \pi R^{2} \cdot\left(\frac{N u_{s t r} k_{a i r}}{2 R}\right) \cdot\left(T_{s}-T_{0}\right)+\dot{m} h_{f g} .
$$

The vaporization rate, for a single droplet is estimated ${ }^{10}$ to be $\dot{m}=2 \pi \rho_{g} D_{i \infty} R \cdot \overline{S h} \ln \left(1+B_{M}\right)$. Here, Spalding mass
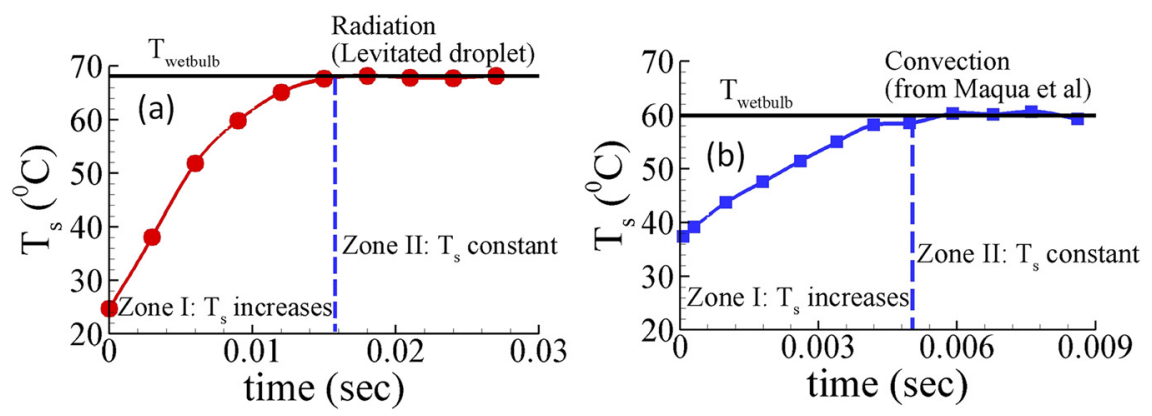

FIG. 1. Temperature rise during heating of ethanol droplet: (a) radiation $\left(0.65 \mathrm{MW} / \mathrm{m}^{2}\right.$ laser flux with $125 \mu \mathrm{m}$ droplet) using IR camera, (b) convection (gas temperature $837^{\circ} \mathrm{C}$ with $105 \mu \mathrm{m}$ droplet) from Maqua et al. (see Ref. 15) (single column). 
transfer number, $B_{M}=\frac{\chi_{g, s}-\chi_{g, \infty}}{1-\chi_{g, s}}$ depends on vapor mass fraction on the droplet surface $\chi_{g, s}$ and vapor mass fraction far away from the droplet surface, $\chi_{g, \infty}$. The vapor mass fraction of the liquid on the droplet surface can be calculated by Raoult's law. ${ }^{10}$ We used the incompressible limit of Sherwood number specified for small droplet. ${ }^{3}$ The average Sherwood number is given by $\overline{S h}=1.89 B / \sqrt{\omega D_{i \infty}}$, where $\omega$ is the angular frequency of the levitator (frequency of the transducer in the levitator) and $D_{i \infty}$ is the mass diffusion coefficient of the liquid vapor into air, and B is the velocity scale which depends on the levitator sound pressure level (SPL) and frequency. ${ }^{3}$

Equation (4) is used to calculate the temperature $\left(T_{\mathrm{s}}\right)$ at small time steps until it converges [99\% rule] and satisfies the equilibrium condition given in Eq. (5). We denote this equilibrium temperature as $\mathrm{T}_{\mathrm{f}-\mathrm{rad}}$, which is the desired temperature scale for radiation. To calculate the equilibrium time scale $\left(\mathrm{t}_{\mathrm{s}-\mathrm{rad}}\right)$ required by the droplet to reach $\mathrm{T}_{\mathrm{f}-\mathrm{rad}}$, a modified form of Eq. (4) (including a correction based on energy spent for vaporization) is used to obtain the expression

$$
t_{s-\text { rad }}=-\frac{1}{C_{1-\text { rad }}} \ln \left[1-\frac{C_{1-r a d}}{C_{2-r a d}}\left(T_{f-r a d}-T_{0}\right)\right] .
$$

To validate the theory and the scaling argument presented above, a set of experiments has been conducted with different liquids and different laser intensities. The experimental setup used an acoustic levitator $(100 \mathrm{kHz}, 156 \mathrm{~dB})$ to suspend the droplet which was irradiated at different intensities by a $\mathrm{CO}_{2}$ laser $(10.6 \mu \mathrm{m}$ wavelength, maximum heat flux of $10 \mathrm{MW} / \mathrm{m}^{2}$ ). A high speed CCD camera along with an IR camera was used for simultaneous imaging. High speed images, captured at 3000-5000 fps (frames per second), were used for calculating droplet diameter reduction rate. The spatial resolution obtained by the zoom lens is approximately $2 \mu \mathrm{m} /$ pixel. An LED (Light Emitting Diode) light source was used to back-illuminate the droplet. The images from the high speed camera were used to determine the instantaneous diameter of the droplet. The process uses intensity gradient to locate edge of the droplet. The uncertainty from this technique was found to be $\pm 4 \mu \mathrm{m}$. IR images, captured at $100-$ $300 \mathrm{fps}$ were used for measuring surface temperature of the droplet. The IR camera is calibrated for a range of $-5-200^{\circ} \mathrm{C}$ with an accuracy of $\pm 1 \%$ of measuring temperature $\left(\sim 50^{\circ} \mathrm{C}\right)$ or $\pm 0.5^{\circ} \mathrm{C}$. The spatial resolution achieved through the zoom lens is $5 \mu \mathrm{m} / \mathrm{pixel}$. The IR camera was operated at 100-300 fps and the recorded images were processed to extract the temperature data of the droplet during the heating process. Further details of the experimental setup are provided in Refs. 4-8. The effective laser flux or $\mathrm{I}_{\mathrm{eff}}$ is less than the actual laser power due to different factors: (a) absorption coefficient, (b) scattering loss, (c) divergence of laser beam. However, except for absorption coefficient, the other factors generally depend on radiation wavelength and geometry of the droplet. With the consideration that the levitated droplets are perfectly spherical in shape, we can assume that these effects are uniform for all liquids when heated with the same monochromatic source. Thus, efficiency, $\eta$, is defined as $I_{\text {eff }}=\eta \cdot I_{0}$. This efficiency term includes the cumulative effect of all the losses. In the current levitation setup, $\eta$ will change only with absorption coefficient of fluids. To obtain the laser efficiency, we performed a calibration experiment using a water droplet at different laser fluxes and determined the efficiency to be $22 \%$. The laser flux efficiency can also change due to small oscillations observed in levitated droplet. The oscillations are generally of the order of $50 \mu \mathrm{m}$. This results in $1 \%-2 \%$ change in droplet location, and we ignored this minor inhomogeneity.

The efficiency $\eta$ mentioned here is based on heat flux, not on laser power. The laser used for this experiment has a $2 \mathrm{~mm}$ beam diameter with the maximum power output of $30 \mathrm{~W}$. This results in a maximum laser flux of around $10 \mathrm{MW} / \mathrm{m}^{2}$. Based on efficiency, $\eta=22 \%$, the maximum effective laser flux for a water droplet is $2.2 \mathrm{MW} / \mathrm{m}^{2}$. However, the corresponding laser power on the droplet depends on droplet size. For a $500 \mu \mathrm{m}$ water droplet, this maximum laser power absorbed by the droplet is $0.43 \mathrm{~W}$ which is approximately $1.5 \%$ of the maximum laser power, $30 \mathrm{~W}$.

Figure 2 shows an increase of surface temperature with time for ethanol droplets. The $125 \mu \mathrm{m}$ droplet with effective laser flux $\left(\mathrm{I}_{\text {eff }}\right)$ of $0.65 \mathrm{MW} / \mathrm{m}^{2}$ undergoes a rapid temperature rise compared to the $500 \mu \mathrm{m}$ droplet with $\mathrm{I}_{\text {eff }}$ of $0.25 \mathrm{MW} / \mathrm{m}^{2}$ [Figs. 2(a) and 2(b)]. The former required around $0.02 \mathrm{~s}$ to attain the wet bulb temperature of $68^{\circ} \mathrm{C}$ while the latter reached a wetbulb temperature of $62^{\circ} \mathrm{C}$ in $0.2 \mathrm{~s}$. The figures also show the theoretical temperature rise (using Eq. (4)) and the theoretical wetbulb temperature, $\mathrm{T}_{\mathrm{f} \text {-rad }}$, using Eq. (5). The intersection of these two curves denotes the equilibrium time scale, $t_{\text {s-rad. }}$. The theoretical analysis shows the correct order of magnitude of the timescale and almost exact wetbulb temperature $\left(\mathrm{T}_{\mathrm{f}-\mathrm{rad}}\right)$ when compared to the experimental data. The normalized temperature $\mathrm{T}^{*}\left(T^{*}=\left(T_{s}-T_{0}\right) /\left(T_{f-\mathrm{rad}}-T_{0}\right)\right)$ vs $t^{*}\left(t^{*}=t / t_{s-\mathrm{rad}}\right)$ for the two drop sizes show excellent agreement with the experimental data.

Next, in order to obtain a timescale for convective heating and establish equivalence with radiative heating, we invoke some of the theoretical framework developed in the literature for a convectively vaporizing droplet and spray. ${ }^{10,11}$ For droplet with small diffusion time scale, one can ignore the thermal gradient within the droplet [Biot
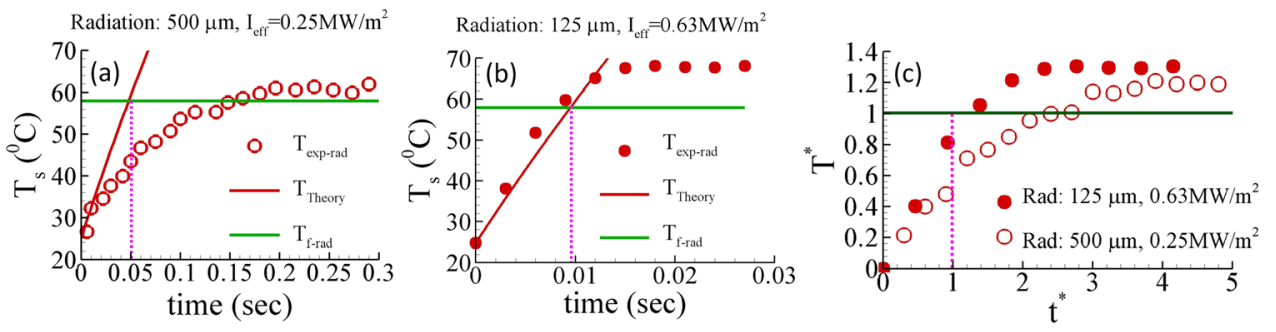

FIG. 2. Ethanol: $\mathrm{T}_{\mathrm{s}}\left({ }^{\circ} \mathrm{C}\right)$ vs time (s) (a) $125 \mu \mathrm{m}$ droplet, $\mathrm{I}_{\text {eff }}=0.25 \mathrm{MW} / \mathrm{m}^{2}$. (b) $500 \mu \mathrm{m}, \mathrm{I}_{\mathrm{eff}}=0.63 \mathrm{MW} / \mathrm{m}^{2}$, (c) $\mathrm{T}^{*} \mathrm{vs} \mathrm{t} \mathrm{t}^{*}$ for these two cases (double column). 


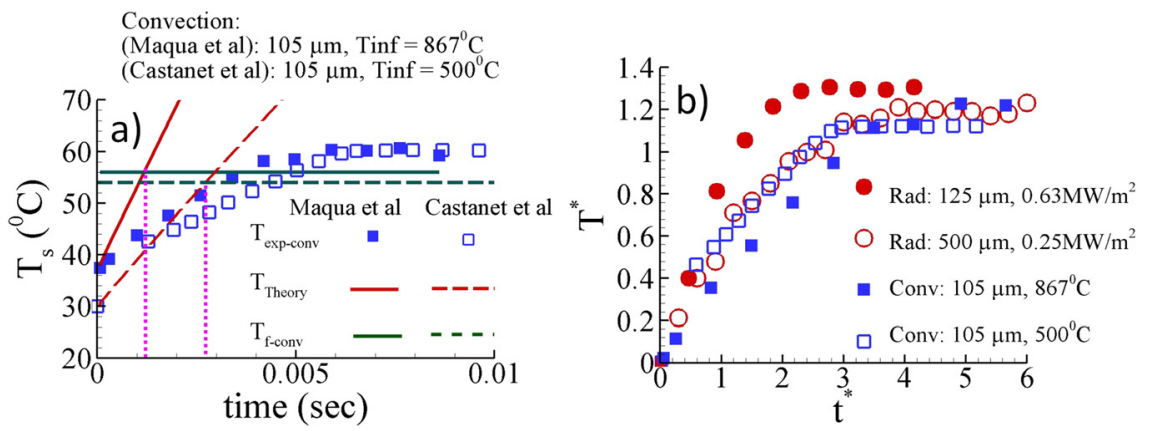

FIG. 3. Ethanol: $\mathrm{T}_{\mathrm{s}}\left({ }^{\circ} \mathrm{C}\right)$ vs time (s) (a) $105 \mu \mathrm{m}$ droplet Castanet et al. (Ref. 16) and Maqua et al. (Ref. 15). (b) Normalized data ( $\mathrm{T}^{*} \mathrm{vs} \mathrm{t}^{*}$ ) for both radiative and convective experiments (double column).
Number $<0.1]$. Following the previous analysis, it can be concluded that the vaporization rate at the initial stages [Zone I in Fig. 1] is negligible. Hence the heat balance equation becomes

$$
h_{c} \cdot 4 \pi R^{2} .\left(T_{\infty}-T_{s}\right)=\frac{4}{3} \pi R^{3} \rho_{l} C_{p} \frac{d T_{s}}{d t}
$$

$T_{\infty}$ is the ambient hot gas temperature. The heat transfer coefficient $\mathrm{h}_{\mathrm{c}}$ is calculated as $h_{c}=N u^{*} k_{\text {air }} / 2 R$. We assume Lewis number to be equal to 1 . Nusselt or Sherwood number for a vaporizing droplet is calculated using Sirignano's model. ${ }^{10}$ The Nusselt and the Sherwood number are both functions of droplet Reynolds number and on the Spalding heat and mass transfer numbers when surface blowing effect is included. For the current analysis, the Reynolds number has been considered to be 10 . This is a good assumption because our analysis focuses on the initial period of heating when the relative velocity between the gas and the droplet is highest.

Equation (7) is solved to yield

$$
T_{s}(t)=T_{0}+\frac{C_{2-\mathrm{rad}}}{C_{1-\mathrm{rad}}}\left[1-\exp \left(-C_{1-\mathrm{rad}} t\right)\right],
$$

where $C_{1-\text { conv }}=\left(\frac{3 N u^{*} k_{\text {air }}}{R^{2} \rho_{l} C_{p}}\right)$ and $C_{2-\text { conv }}=\left(\frac{3 N u^{*} k_{\text {air }}}{R^{2} \rho_{l} C_{p}}\right) \cdot\left(T_{\infty}-T_{0}\right)$. The equilibrium temperature of a droplet in a convective field can be calculated using Sirignano's model ${ }^{10}$ of isolated droplet. Under this assumption, at equilibrium temperature, the droplet should satisfy the equation (9): ${ }^{10}$

$$
\chi_{g, s}=\frac{C_{p, a i r}\left(T_{\infty}-T_{f-\text { conv }}\right)}{h_{f g}+C_{p, a i r}\left(T_{\infty}-T_{f-c o n v}\right)},
$$

where $\mathrm{C}_{\mathrm{p} \text {,air }}$ is the specific heat of air and $\chi_{g, s}$ is the mass fraction of vapor phase on droplet surface, which can be calculated based on Sirignano's model. ${ }^{10}$ An iterative process has been used to determine the equilibrium temperature of the droplet, $\mathrm{T}_{\mathrm{f} \text {-conv }}$. The equilibrium time scale to reach this temperature has been calculated by using a modified form of Eq. (8) similar to the radiation counterpart

$$
t_{s-c o n v}=-\frac{1}{C_{1-c o n v}} \ln \left[1-\frac{C_{1-\text { conv }}}{C_{2-\text { conv }}}\left(T_{f-\text { conv }}-T_{0}\right)\right] .
$$

The proposed scaling analysis for convective heating of droplets was validated against the experimental data of Refs. 15 and 16 for ethanol droplet evaporation in hot air using a monodisperse droplet stream. For the purpose of analysis,
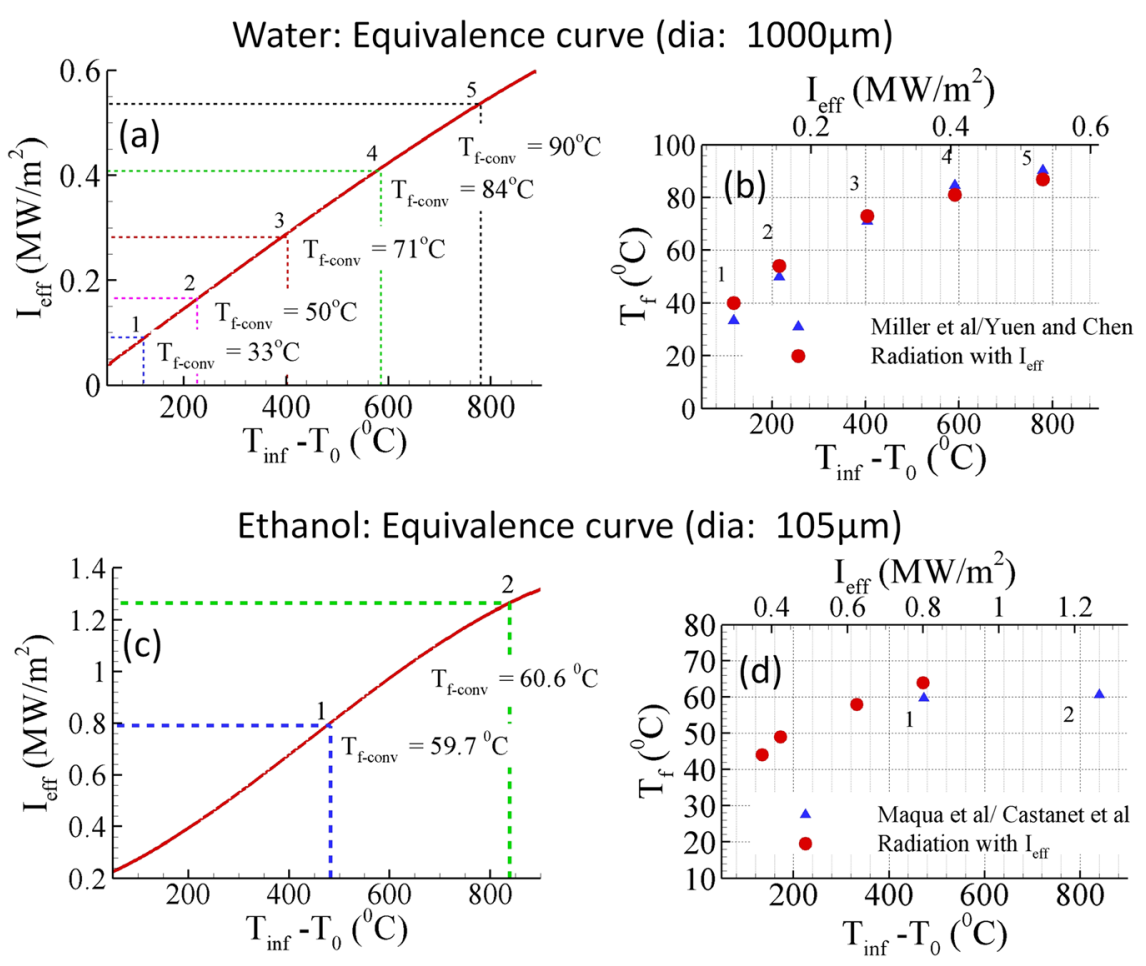

FIG. 4. Equivalent laser flux corresponding to different gas phase temperatures [(a): water $(1000 \mu \mathrm{m})$ and $(\mathrm{c})$ : ethanol $(105 \mu \mathrm{m})]$, Wet bulb temperature achieved in experiments using certain gas phase temperature and equivalent laser flux [(b): water $(1000 \mu \mathrm{m})$ and $(\mathrm{d})$ : ethanol $(105 \mu \mathrm{m})]$ (convection data are taken from Refs. 12, 13, 15, and 16) (double column). 
we considered a constant gas phase temperature of $500^{\circ} \mathrm{C}$ in experiments. ${ }^{16} \mathrm{We}$ also validated against Maqua et al's data [Ref. 15] in a flame environment where the gas phase temperature was as high as $867^{\circ} \mathrm{C}$. These data are shown in Fig. 3(a). Figure 3 also contains the theoretical profile for temperature rise (Eq. (8)) and theoretical wet bulb temperature, $\mathrm{T}_{\mathrm{f} \text {-conv }}$ (Eq. (9)). The intersection of these two curves yields the equilibrium time scale $\left(\mathrm{t}_{\mathrm{s}-\mathrm{conv}}\right)$. There is excellent agreement with the experimental data with respect to the equilibrium temperature and the equilibrium timescale. The normalized profiles ( $\mathrm{T}^{*}$ vs. $\mathrm{t}^{*}$, where for radiative: $T^{*}=$ $\left(T_{s}-T_{0}\right) /\left(T_{f-\text { rad }}-T_{0}\right)$ and $t^{*}=t / t_{s-\text { rad }}$ and for convective $T^{*}=\left(T_{s}-T_{0}\right) /\left(T_{f-\text { conv }}-T_{0}\right)$ and $\left.t^{*}=t / t_{s-\text { conv }}\right)$ also show excellent unification of the two entirely different sets of data (Fig. 3(b)) for both radiative and convective heating, for different droplet sizes, gas phase temperature, and laser flux.

The scaling analysis not only shows that the data from the two modes of heating can be unified but also provides the required laser flux $\left(\mathrm{I}_{\mathrm{eff}}\right)$ to simulate a certain convective condition. Using Eq. (9), the wetbulb temperature scale $\left(\mathrm{T}_{\mathrm{f} \text {-conv }}\right)$ for a certain size of the droplet of a fluid can be calculated at a given $\left(\mathrm{T}_{\mathrm{inf}}-\mathrm{T}_{0}\right)$. Now, using Eq. (5) for the same droplet diameter, one can estimate required $\mathrm{I}_{\text {eff }}$ for irradiated droplet to reach the same wetbulb temperature scale $\left(\mathrm{T}_{\mathrm{f}-\mathrm{rad}}\right)$. Figures $4(\mathrm{a})$ and $4(c)$ show the variation of equivalent $I_{\text {eff }}$ with $\left(T_{\text {inf }}-T_{0}\right)$ for $1 \mathrm{~mm}$ water droplet and $105 \mu \mathrm{m}$ ethanol droplet. Five experimental convective conditions for water and two for ethanol have been reported. ${ }^{12,13,15,16}$ The wetbulb temperatures for convection and radiation experiments [Figs. 4(b) and 4(d)] compare favorably using equivalent $\mathrm{I}_{\text {eff. }}$ The minimum practical ethanol dropsize that could be levitated was $125 \mu \mathrm{m}$ in the radiation experiment that could be compared with the $105 \mu \mathrm{m}$ drop $^{16}$ in the convection experiment.

Time and temperature scales are developed in this letter, which can be applied to both convective heating and radiative heating of liquid droplets. The transient temperature profiles normalized by these scales show a single unified profile irrespective of the heating mode, liquid properties, and external heating conditions. A much needed connection between a single stream of convectively heated droplet and irradiated levitated droplets has been established to obtain the wet bulb temperature. A theoretical framework has also been provided to match the laser flux to obtain the wet bulb temperature in an equivalent convective environment at a given gas phase temperature.

${ }^{1}$ W. J. Xie, C. D. Cao, Y. J. Lu, and B. Wei, Phys. Rev. Lett. 89, 104304 (2002).

${ }^{2}$ W. J. Xie, C. D. Cao, Y. J. Lü, Z. Y. Hong, and B. Wei, Appl. Phys. Lett. 89, 214102 (2006).

${ }^{3}$ A. L. Yarin, G. Brenn, O. Kastner, D. Rensink, and C. Tropea, J. Fluid Mech. 399, 151 (1999).

${ }^{4}$ A. Saha, S. Basu, and R. Kumar, Int. J. Heat Mass Trans. 53, 3862 (2010).

${ }^{5}$ S. Basu, A. Saha, and R. Kumar, Appl. Phys. Lett. 100, 054101 (2012).

${ }^{6}$ R. Kumar, E. Tijerino, A. Saha, and S. Basu, Appl. Phys. Lett. 97, 123106 (2010).

${ }^{7}$ A. Saha, S. Basu, and R. Kumar, Exp. Fluids 52, 795 (2012).

${ }^{8}$ A. Saha, S. Basu, C. Suryanarayana, and R. Kumar, Int. J. Heat Mass Trans. 53, 5663 (2010).

${ }^{9}$ A. Saha, V. Singh, S. Seal, and S. Basu, Surf. Coat. Technol. 203, 2102 (2009).

${ }^{10}$ W. A. Sirignano, Fluid Dynamics and Transport of Droplets and Sprays, 2nd ed. (Cambridge University Press, New York, 2010).

${ }^{11}$ C. K. Law, Prog. Energy Combust. Sci. 8, 171 (1982).

${ }^{12}$ R. S. Miller, K. Harstad, and J. Bellan, Int. J. Multiphase Flow 24, 1025 (1998).

${ }^{13}$ M. C. Yuen and L. W. Chen, Combust. Sci. Technol. 14, 147 (1976).

${ }^{14}$ A. Daif, M. Bouaziz, X. Chesneau, and A. A. Cherif, Exp. Therm. Fluid Sci. 18, 282 (1998).

${ }^{15}$ C. Maqua, G. Castanet, F. Grisch, F. Lemoine, T. Kristyadi, and S. S. Sazhin, Int. J. Heat Mass Trans. 51, 3932 (2008).

${ }^{16}$ G. Castanet, P. Lavieille, F. Lemoine, M. Lebouche, A. Atthasit, Y. Biscos, and G. Lavergne, Int. J. Heat Mass Trans. 45, 5053 (2002).

${ }^{17}$ G. Castanet, M. Lebouché, and F. Lemoine, Int. J. Heat Mass Trans. 48, 3261 (2005)

${ }^{18}$ A. L. Yarin, M. Pfaffenlehner, and C. Tropea, J Fluid Mech. 356, 65 (1998).

${ }^{19}$ A. Saha, S. Basu, and R. Kumar, J. Fluid Mech. 692, 207 (2012).

${ }^{20}$ B. H. Park and R. L. Armstrong, Appl. Opt. 28, 3671 (1989).

${ }^{21}$ L. Dombrovsky and S. S. Sazhin, Int. J. Heat Fluid Flow 24, 919 (2003).

${ }^{22}$ S. Basu and B. M. Cetegen, J. Heat Trans. 130, 501 (2008). 